\section{ÜBRIGENS}

... schätzt das Nationale Referenzzentrum für Borrelien in Bayern, dass in Deutschland iedes Jahr 60.000 bis 100.000

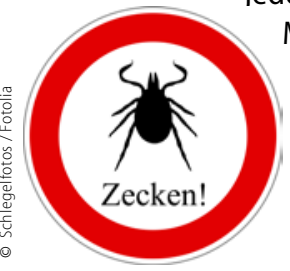
Menschen neu an durch Zecken übertragene LymeBorreliose erkranken. Gesicherte Zahlen gibt es nicht, da keine einheitliche Meldepflicht besteht.

... werden bei $75 \%$ der in Deutschland gemeldeten Infektionen mit dem Dengue-Virus asiatische Länder als Infektionsland genannt, meist Thailand.

... soll der Malaria-Impfstoff RTS,S (Mosquirix $^{\mathrm{TM}}$ ) in Ghana, Kenia und Malawi ab dem Jahr 2018 auf Alltagstauglichkeit getestet werden, wie die WHO meldet. Der Impfstoff soll Kinder

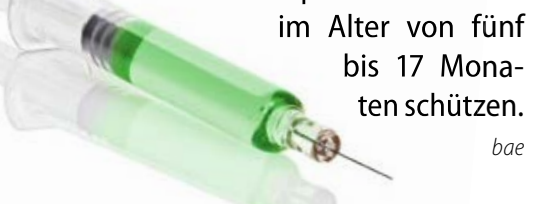

Punkte sammeln in der Sommerakademie mit e.Med

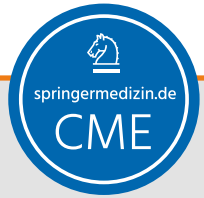

Auch 2017 haben wir in der Sommerakademie ausgewählte CME-Kurse für Ihre hausärztliche Fortbildung zusammengestellt.

Zum Thema "Reisemedizin" finden Sie unter http://bit.ly/2hFwgdK

aktuell den Kurs

„Eine migrationsmedizinische Heraus-

forderung? Die ambulante Versorgung Geflüchteter"

Zur Teilnahme benötigen sie ein e.Med-Abo, mit dem sie Zugang zu allen Inhalten auf SpringerMedizin.de erhalten.

Nutzen Sie die Sommerzeit, um CME-Punkte zu sammeln und um sich schnell und effizient fortzubilden. Testen Sie SpringerMedizin.de 30 Tage lang kostenlos und unverbindlich mit dem e.Med-Abo: http://bit.ly/2vhm8el

FSME

\title{
Urlaub mit Zeckenschutz planen
}

2016 hat die Zahl der FSME-Infektionen im Vergleich zum Vorjahr um $59 \%$ zugenommen.

Die FSME-Fallzahlen sind im Jahr 2016 im Vergleich zum Vorjahr um 59\% gestiegen. Das gibt das Robert Koch-Institut (RKI) in seinem kürzlich veröffentlichten Epidemiologischen Bulletin zu den FSME-Risikogebieten in Deutschland an. Als Infektionsland wurde dabei in 309 der insgesamt 348 an das RKI übermittelten Fälle nur Deutschland genannt. Gemeldet wurden aber auch Infektionen aus Spanien, Frankreich, Lettland, Österreich, Polen und der Russischen Föderation. Die hiesigen Risikogebiete sind wie im vergangenen Jahr 146 Kreise, hauptsächlich betroffen sind Bayern mit 83 und Baden-Württemberg mit 43 Kreisen.

Erstmals wurde 2016 in Deutschland eine FSME-Erkrankung durch den Verzehr von infizierter Ziegenmilch belegt: Zwei Menschen erkrankten, weil einige
Rohmilchprodukte eines Hofs mit FSMEViren verunreinigt waren. Eine Übertragung der Viren durch infizierte Milch von Schafen und Ziegen ist jedoch nach Angaben des RKI sehr selten. Noch unwahrscheinlicher sei eine Übertragung durch Kuhmilch.

\section{Schutz durch Impfung}

Zum ersten Mal wurden FSME-Viren auch in Auwaldzecken nachgewiesen, die bisher nicht als Überträger des FSMEVirus galten. Ob die Auwaldzecke erst kürzlich zum Überträger wurde oder ob ihre Gefährlichkeit bisher nicht bekannt war, ist Experten zufolge unklar.

Der zuverlässigste Schutz gegen FSME ist die Schutzimpfung. Die Ständige Impfkommission empfiehlt sie generell für Personen ab drei Jahren, die in Risikogebieten wohnen oder arbeiten und dabei ein Risiko für Zeckenstiche haben. Auch Personen, die sich aus anderen Gründen in Risikogebieten aufhalten und zeckenexponiert sind, sollten sich impfen lassen. bae

\section{Pneumokokken}

\section{Impf-Empfehlung besser umsetzen!}

Trotz des hohen Risikos für eine Pneumokokken-Pneumonie sind nur wenige Senioren geimpft. Die STIKO appelliert daher für eine bessere Umsetzung der Impf-Empfehlungen.

Gerade bei der größten Risikogruppe den Senioren ab 60 Jahren - ist die Pneumokokken-Impfrate besonders niedrig: Der Versorgungsatlas des Zentralinstituts für die kassenärztliche Versorgung in Deutschland gibt für die Jahre 2010 bis 2014 bei den 60-64-Jährigen eine kumulierte Durchimpfungsrate von 10,2\% an. „Eine bessere Umsetzung der Impf-Empfehlungen ist dringend wünschenswert", betont die STIKO daher im Epidemiologischen Bulletin zur Aktualisierung der Pneumokokken-Impfempfehlung für Senioren, das Ende 2016 erschienen ist.

Darin empfiehlt die STIKO nun für Personen ab 60 Jahren die alleinige Impfung mit dem 23-valenten Polysaccharid-
Impfstoff PPSV23 (Pneumovax ${ }^{\oplus 23) . ~ E r-~}$ gänzend zu bisherigen Empfehlungen rät die STIKO zudem zur PneumokokkenImpfung mit PPSV23 für Personen mit beruflichen Tätigkeiten wie Schweißen und Trennen von Metallen. Neu ist auch der Rat für Wiederholungsimpfungen: Aufgrund der begrenzten Dauer des Impfschutzes sollten weitere Impfungen im Abstand von sechs Jahren erfolgen.

Der 13-valente Konjugat-Impfstoff PCV13 (Prevenar13 ${ }^{\circ}$ ) war vor einigen Jahren ein Durchbruch für die Impfung von Säuglingen und Kleinkindern: Erstmals ließ sich damit ein Pneumokokken-Schutz bei einem unreifen Immunsystem erzielen. Seit 2015 ist PCV13 auch für die Prävention einer Pneumokokken-Pneumonie bei Erwachsenen ab 18 Jahren zugelassen. Bei Hochrisikopatienten empfiehlt die STIKO eine sequenzielle Pneumokokken-Impfung mit PCV13 gefolgt von PPSV23 nach sechs bis zwölf Monaten. 